\title{
Precision spectroscopy of exotic atoms involving muons
}

\author{
Sohtaro Kanda* \\ RIKEN \\ E-mail: sohtaro.kandaeriken.jp \\ Katsuhiko Ishida \\ RIKEN \\ E-mail: ishidadriken.jp \\ Koichiro Shimomura \\ KEK \\ E-mail: ksimomuepost.kek.jp
}

Precision spectroscopy of hydrogen-like atoms is a powerful method to study fundamental interactions and constants. In particular, exotic atoms involving muons are useful for a precision test of bound-state QED theory and a search for physics beyond the Standard Model. Several precision spectroscopy projects of muonic systems are in progress at J-PARC, where the highest-intensity pulsed muon beam is provided. The MuSEUM collaboration aims to measure the ground-state hyperfine splitting (HFS) of muonium and determine the muon-to-electron mass ratio. The first phase of the experiment has been started and the microwave resonance of the muonium HFS was successfully observed. Toward solving an unsolved problem in the proton charge radius, laser spectroscopy of muonic hydrogen was newly proposed to measure the ground-state HFS and determine the proton Zemach radius. This experiment is in preparation and a feasibility study was performed.

The 20th International Workshop on Neutrinos (NuFact2018)

12-18 August 2018

Blacksburg, Virginia

${ }^{*}$ Speaker. 


\section{Precision spectroscopy of hydrogen-like atoms}

Advances in laser spectroscopy realized an excellent precision of the transition frequencies. Meanwhile, the precision of the theoretical predictions was relatively limited since the proton is a composite particle and the understanding of its internal structure is limited. Spectroscopy of exotic atoms gives distinctive opportunities to study physics of two-body systems. Following sections will describe two exotic hydrogen-like atoms involving muon: muonium and muonic hydrogen. The former is an ideal system for a rigorous test of bound-state quantum electrodynamics (QED) theory because it is not involving proton. The latter gives valuable information about the internal structure of the proton.

\section{Microwave spectroscopy of muonium}

Muonium is a bound state of a positive muon and an electron. In the standard model of particle physics, muonium is a two-body system of structureless leptons. Precision measurement of the ground-state hyperfine splitting (HFS) in muonium is the most stringent validation of bound-state QED theory and the most precise method to determine the muon-to-electron mass ratio.

The most precise value of the muonium HFS had been obtained by an experiment at Los Alamos Meson Physics Facility (LAMPF) [四]. Measurement precision was statistically limited because the trigger rate was restricted to avoid muon beam pileup. This limitation is an intrinsic difficulty with continuous muon beams. At Japan Proton Accelerator Research Complex (J-PARC), a new precision measurement with high-intensity pulsed muon beam was proposed. An experiment with a pulsed beam is advantageous in statistics. However, this benefit involves another difficulty in signal detection due to a high instantaneous event rate. To overcome this obstacle, a high-rate capable positron counter was developed [ [2].

The muonium HFS can be measured by two independent methods; a direct measurement at zero magnetic field and an indirect one utilizing the Zeeman splitting in a high magnetic field. These measurements are complementary from the viewpoint of systematic uncertainties and sensitivities to physics beyond the standard model. The MuSEUM collaboration aims to improve the statistical precision by a factor of ten for both methods.

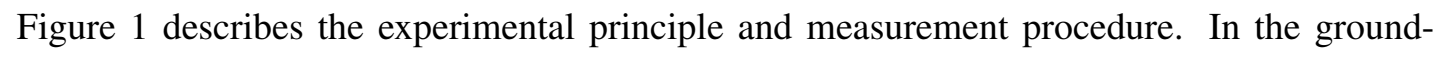
state, muonium is in the spin-singlet or the spin-triplet states. A hyperfine transition occurs under microwave irradiation having a frequency of $4.463 \mathrm{GHz}$. The hyperfine state can be determined as an ensemble average by measurement of decay positron's angular asymmetry (Fig.마a). The muon spin flips following the hyperfine transition as a function of time (Fig.마). The transition probability depends on the microwave frequency detuning from the HFS (Fig.tI-c).

Since the experiment was proposed in 2011 []], apparatus developments and construction of the dedicated beamline have been pursued. In 2016, a measurement at zero magnetic field resulted in the first observation of the HFS resonance [ [ 9 ]. Improvements in the zero-field measurement and preparations for a high field measurement are in progress.

\section{Laser spectroscopy of muonic hydrogen}

Muonic hydrogen is a hydrogen-like atom consisting of a negative muon and a proton. Its Bohr radius is 200 times smaller than that of electronic hydrogen. Therefore, spectroscopy of muonic 
(a)

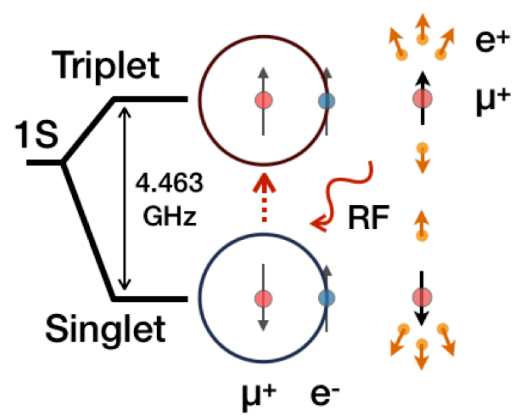

(b)

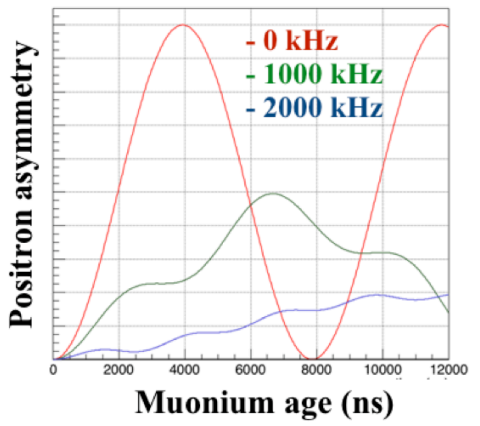

(c)

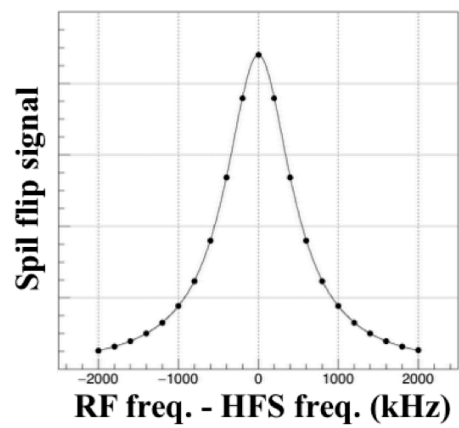

Figure 1: Measurement procedure of the muonium HFS spectroscopy: (left) hyperfine structure of muonium; (center) analytically calculated time-dependent positron asymmetry arising from a hyperfine transition; (right) numerically simulated lineshape of the HFS resonance.

hydrogen is highly sensitive to the internal structure of the proton via the finite volume effect of a composite particle. At Paul Scherrer Institute (PSI), the proton charge radius was determined via laser spectroscopy of the Lamb shifts in muonic hydrogen atom [ [ $[$, 目]. The experiment observed a significantly discrepant result compared to the ones by electronic hydrogen spectroscopy and electron-proton scattering [ $[0,8]$. The anomaly is known as "proton radius puzzle" and it has been unsolved even after various theoretical and experimental efforts.

Alternatively, the structure of proton is expressed by the Zemach radius, which is defined as a convolution of the electric charge and magnetic moment distributions. The Zemach radius contributes linearly to the shift of the HFS. In contrast to the case of the charge radius, the determination precision of the Zemach radius by the muonic hydrogen spectroscopy at PSI was insufficient to discuss the consistency between other measurements. This is because the muonic result was indirectly derived from two Lamb shift frequencies via the $2 \mathrm{~S}$ hyperfine splitting. To shed some light on the proton radius puzzle, a new measurement of the ground-state HFS in muonic hydrogen was proposed. The experiment will be performed with the high-intensity pulsed muon beam at J-PARC.

The measurement principle and procedure are essentially equal to ones for the muonium HFS spectroscopy. For the case of muonic hydrogen HFS, the transition frequency is $44.2 \mathrm{THz}$ and a mid-infrared laser will be employed to induce the hyperfine transition. After the proposal submission in 2014, a transition laser, a cryogenic hydrogen gas target, and an electron detector have been developed. Towards a realization of the spectroscopy experiment, a feasibility test was performed at RIKEN-RAL muon facility and data analysis is underway [Q].

\section{References}

[1] W. Liu et al., Phys. Rev. Lett. 82711 (1999).

[2] S. Kanda et al., Proceedings of Science PoS(PhotoDet2015)036 (2016).

[3] K. Shimomura, AIP Conf. Proc. 1382245 (2011).

[4] S. Kanda for the MuSEUM Collaboration, Proceedings of Science PoS(INPC2016)170 (2017).

[5] R. Pohl et al., Nature 466213 (2010).

[6] A. Antognini et al., Science 339417 (2013).

[7] P. J. Mohr, D. B. Newell, and B. N. Taylor, Rev. Mod. Phys. 88035009 (2016).

[8] J. C. Bernauer et al., Phys. Rev. C 90015206 (2014).

[9] S. Kanda et al., J. Phys.: Conf. Ser. 1138012009 (2018). 